\title{
Strain development during the phase transition of $\mathrm{La}(\mathrm{Fe}, \mathrm{Mn}, \mathrm{Si}) 13 \mathrm{~Hz}$
}

Neves Bez, Henrique; Nielsen, Kaspar Kirstein; Smith, Anders; Norby, Poul; Ståhl, Kenny; Bahl, Christian R H

Published in:

Applied Physics Letters

Link to article, DOI:

$10.1063 / 1.4960358$

Publication date:

2016

Document Version

Publisher's PDF, also known as Version of record

Link back to DTU Orbit

Citation (APA):

Neves Bez, H., Nielsen, K. K., Smith, A., Norby, P., Ståhl, K., \& Bahl, C. R. H. (2016). Strain development during the phase transition of $\mathrm{La}(\mathrm{Fe}, \mathrm{Mn}, \mathrm{Si}){ }_{13} \mathrm{H}_{z}$. Applied Physics Letters, 109(5), [051902].

https://doi.org/10.1063/1.4960358

\section{General rights}

Copyright and moral rights for the publications made accessible in the public portal are retained by the authors and/or other copyright owners and it is a condition of accessing publications that users recognise and abide by the legal requirements associated with these rights.

- Users may download and print one copy of any publication from the public portal for the purpose of private study or research.

- You may not further distribute the material or use it for any profit-making activity or commercial gain

- You may freely distribute the URL identifying the publication in the public portal

If you believe that this document breaches copyright please contact us providing details, and we will remove access to the work immediately and investigate your claim. 


\title{
Strain development during the phase transition of $\mathrm{La}(\mathrm{Fe}, \mathrm{Mn}, \mathrm{Si})_{13} \mathrm{H}_{z}$
}

\author{
Henrique Neves Bez, ${ }^{1, a)}$ Kaspar K. Nielsen, ${ }^{1}$ Anders Smith, ${ }^{1}$ Poul Norby, ${ }^{1}$ Kenny Ståhl, ${ }^{2}$ \\ and Christian R. H. Bahl ${ }^{1}$ \\ ${ }_{1}^{1}$ Department of Energy Conversion and Storage, Technical University of Denmark, Frederiksborgvej 399, \\ DK-4000 Roskilde, Denmark \\ ${ }^{2}$ Department of Chemistry, Technical University of Denmark, Anker Engelunds Vej, DK-2800 Lyngby, \\ Denmark
}

(Received 27 April 2016; accepted 22 July 2016; published online 1 August 2016)

\begin{abstract}
We use powder X-ray diffraction to evaluate the temperature dependence of the crystalline properties during the magnetic phase transition of $\mathrm{La}\left(\mathrm{Fe}, \mathrm{Mn}, \mathrm{Si}_{13} \mathrm{H}_{z}\right.$ as a function of the $\mathrm{Fe} / \mathrm{Mn} / \mathrm{Si}$ ratio. Both the paramagnetic and ferromagnetic phases were observed as peak overlaps in the patterns around the Curie temperature $\left(T_{\mathrm{C}}\right)$ occurring continuously in a temperature range of about $5 \mathrm{~K}$ around $T_{\mathrm{C}}$. Using the Williamson-Hall method, we evaluate the strain developing in the crystallites during the transition and find that it is associated with the growth of the paramagnetic phase as the transition occurs. Based on our measurements and microstructure analyses, we propose that cracking during the phase transition is due to or aggravated by the small content of a La-rich phase. Published by AIP Publishing. [http://dx.doi.org/10.1063/1.4960358]
\end{abstract}

Ferromagnetic (FM) materials exhibit the magnetocaloric effect to a greater or lesser extent: A change in temperature when an external magnetic field is varied adiabatically, the effect being largest close to the Curie transition of the material. Materials with a sizable magnetocaloric effect close to room temperature have attracted particular interest in recent years due to their possible application for refrigeration. ${ }^{1}$ Such materials can be classified as either (i) first order phase transition (FOPT) materials, which exhibit latent heat during the transition and where the transition usually involves structural and/or volume change or (ii) second order phase transition (SOPT) materials, which do not have latent heat associated with the transition nor structural or volume change. In general, FOPT materials can be associated with a large magnetocaloric effect but also with hysteresis losses.

One much-studied material series that can have a FOPT is $\mathrm{La}(\mathrm{Fe}, \mathrm{Mn}, \mathrm{Si})_{13} \mathrm{H}_{z}$, where the Curie temperature, $T_{\mathrm{C}}$, can be controlled by the proportion of $\mathrm{Fe} / \mathrm{Mn} / \mathrm{Si}$. Recently, we have shown by X-ray diffraction (XRD) measurements that for stoichiometries with $T_{\mathrm{C}}>300 \mathrm{~K}$, the materials have a volume change at the phase transition larger than $0.8 \%$ without changing the symmetry of the crystal structure, ${ }^{2}$ as is the case for other La-Fe-Si hydrides. ${ }^{3}$ Even though these materials have a FOPT, the transition is not discontinuous but takes place over a finite temperature range. We further showed that this behavior can be explained within the framework of the Bean-Rodbell model ${ }^{4}$ as being due to chemical inhomogeneities, which effectively lead to a spread of $T_{\mathrm{C}}$ throughout the sample. ${ }^{2}$

Due to the volume change during the phase transition, one can expect that internal stresses will develop. Indeed, samples may break apart during the phase transition, due to the so-called virgin effect..$^{5}$ Moreover, this breaking apart into fragments changes the magnetic signature of the transition, since the coupling between the fragments is weakened. ${ }^{6}$

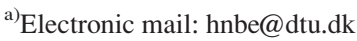

To investigate this in greater detail, we used X-ray diffraction (XRD) measurements in combination with the Williamson-Hall method to determine the strain in both the paramagnetic (PM) and ferromagnetic (FM) phases across the phase transition. The Williamson-Hall $\operatorname{method}^{7}$ analyses the peak broadening, $\beta$, from an XRD pattern in terms of the strain and particle size of the sample

$$
\beta(\theta) \cos \theta=\frac{k \lambda}{D}+4 \epsilon \sin \theta,
$$

where $k$ is a constant, $\lambda$ is the wavelength of the incident radiation, $D$ is a measure of the particle size, $\epsilon$ is the strain, and $\theta$ is the scattering angle. It should be noted that the Williamson-Hall method cannot in general be relied on to get absolute values of the strain. ${ }^{8}$ However, one may deduce relative trends for the strain of the same sample under different external conditions (in the present case temperature).

The material used in this investigation is particulate $\mathrm{LaFe}_{13-x-y} \mathrm{Mn}_{x} \mathrm{Si}_{y} \mathrm{H}_{1.65}$ with $x=0.25,0.22$, and 0.06 and $y=1.28,1.23$, and 1.18 , respectively. This material was synthesized by a powder metallurgical process described elsewhere. ${ }^{9}$ Scanning electron microscopy (SEM) backscattered images were taken in a Hitachi TM3000 microscope equipped with a Bruker Quantax EDS detector. The XRD measurements were performed as explained elsewhere. ${ }^{2}$ SEM images of particles that have undergone XRD measurements showed that the vast majority of particles are below $60 \mu \mathrm{m}$. Rietveld refinement was done in order to extract the peak broadening for each sample at each temperature. To evaluate the effect of the interstitial hydrogen on the strain of the material, a dehydrogenation process was additionally performed on the material with $x=0.06$ : the powder material was heated to $773 \mathrm{~K}$ at $5 \mathrm{Kmin}^{-1}$ under high vacuum; the temperature was held for $1 \mathrm{~h}$ and then slowly cooled to room temperature in the furnace. The dehydrogenated sample did not show extra peaks in the XRD pattern, confirming that no oxide phases were formed during the dehydrogenation process. 


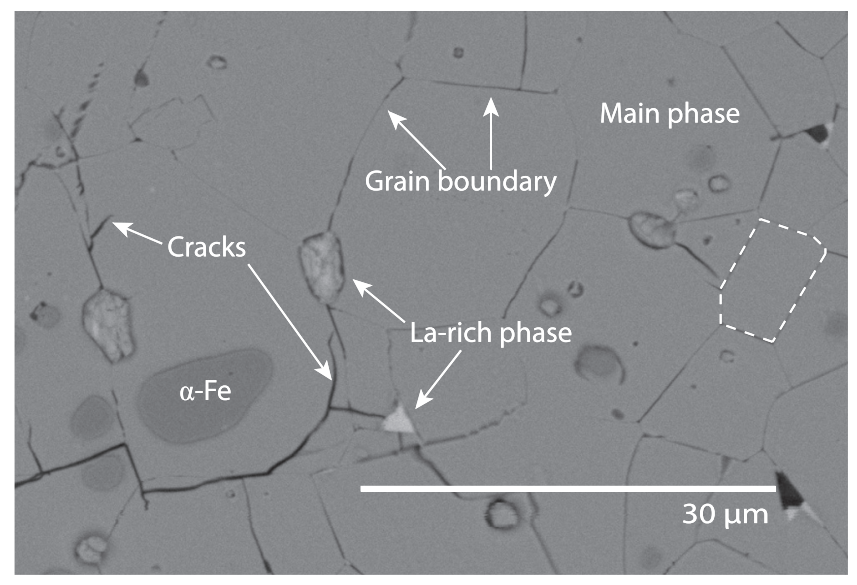

FIG. 1. Particle of $\mathrm{LaFe}_{13-x-y} \mathrm{Mn}_{x} \mathrm{Si}_{y} \mathrm{H}_{1.65}$ where $x=0.06$. One may notice the crystallites with sizes between 15 and $30 \mu \mathrm{m}$, cracks, and small contents of secondary phases, i.e., $\alpha$-Fe and La-rich phase.

Figure 1 shows an SEM backscattered image of a particle, which has undergone XRD measurements. The grain sizes are in the range of 15 to $30 \mu \mathrm{m}$, and $\alpha-\mathrm{Fe}$ is present as a secondary phase together with minor content of a La-rich phase (the white spots in the image). The crack propagation in this particle shows the brittle behavior of the main phase, $\mathrm{La}(\mathrm{Fe}, \mathrm{Mn}, \mathrm{Si})_{13} \mathrm{H}_{z}$. One of the cracks is pointed out in the image starting on the La-rich phase and propagating transgranularly in the main phase. This is the usual crack propagation behavior of a brittle material when the crystal is more brittle than the grain boundary itself. ${ }^{10}$ This is the case for the main phase but not for the ductile secondary phase. It should be noted that the crack did not necessarily develop during the XRD measurements but could be a consequence of the grinding of the particles done prior to the measurement.

Figure 2 shows a color map of the intensity of the XRD patterns for the main peak of $x=0.06$, as a function of temperature and angle. One can observe the co-existence of the FM and PM phases, and the overlapping of the peaks. The inset shows a group of three peaks of the main phase at $340 \mathrm{~K}$ for $x=0.06$. As pure phases, these peaks would be in pairs (due to $K \alpha_{1}$ and $K \alpha_{2}$ radiation); however, due to the lattice parameter difference between the FM and PM phase and the co-existence of both phases, groups of three peaks are observed during the transition.

Usually, the peak broadening $\beta$ is determined by fitting each peak individually. In the present case, such an approach cannot be used since each peak during the phase transition of $\mathrm{La}(\mathrm{Fe}, \mathrm{Mn}, \mathrm{Si})_{13} \mathrm{H}_{z}$ will be the result of an overlap of the two phases. Therefore, it is necessary to apply the Rietveld refinement full-width-at-half-maximum fitting parameters for the analysis. From the refinement, one can express $\beta$ as a function of the angle as given by the Caglioti equation ${ }^{11}$

$$
\beta(\theta)=\left(u \tan ^{2} \theta+v \tan \theta+w\right)^{1 / 2},
$$

where $u, v$, and $w$ are fitting parameters. Instrumental broadening is accounted for through a Gaussian summation of two contributions

$$
\beta^{2}=\beta(\theta)_{\text {measured }}^{2}-\beta(\theta)_{\text {instrumental }}^{2},
$$

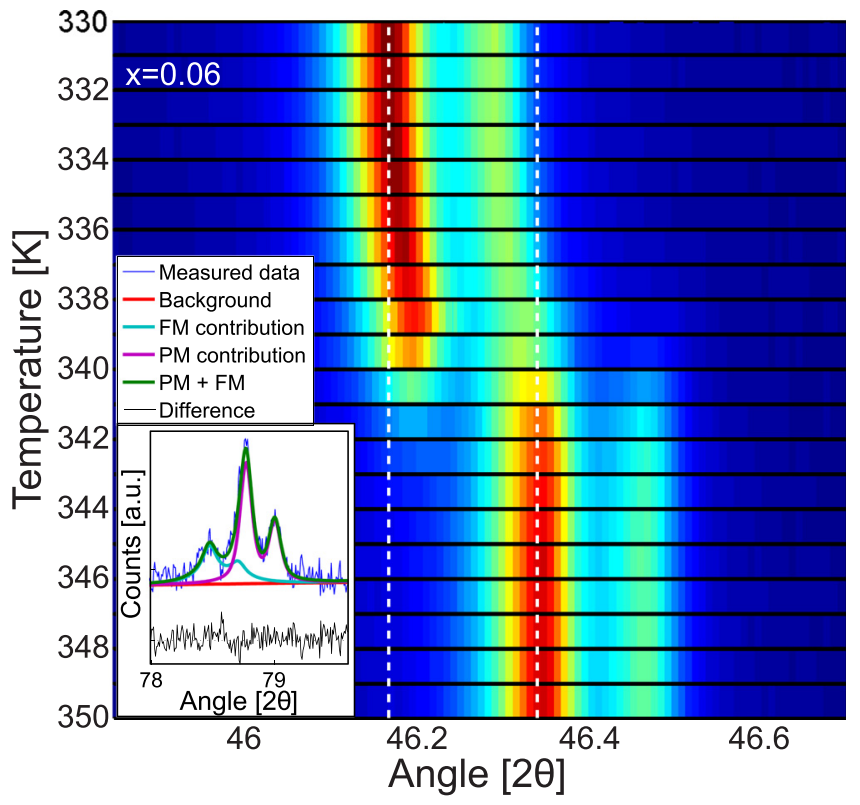

FIG. 2. Zoom at the main pair of peaks across the phase transition. The dashed lines are guides to the eyes. The inset shows a zoom in the peaks (93 1) of the XRD pattern at $340 \mathrm{~K}$.

where $\beta_{\text {instrumental }}$ is extracted from a strain-free standard $\mathrm{LaB}_{6}$ sample, measured under identical conditions. Figure 3 shows the Williamson-Hall plot for the FM phase of $\mathrm{LaFe}_{13-x-y} \mathrm{Mn}_{x} \mathrm{Si}_{y} \mathrm{H}_{1.65}$ where $x=0.06$, for $335 \leq T \leq 341 \mathrm{~K}$. Equation (1) shows that the slope of a curve is in fact the average non-uniform strain at the given temperature. It can be seen that all slopes are positive, which would indicate a positive strain, i.e., that the material is under tensile stress. However, the Williamson-Hall method does not in fact allow a determination of whether the strain is positive or negative. The reason for the peak broadening as an outcome of strain is the non-uniform distances between the atoms in the crystal structure, and both a compressive and a tensile non-uniform strain will give a broadening of the peak. Therefore, we have

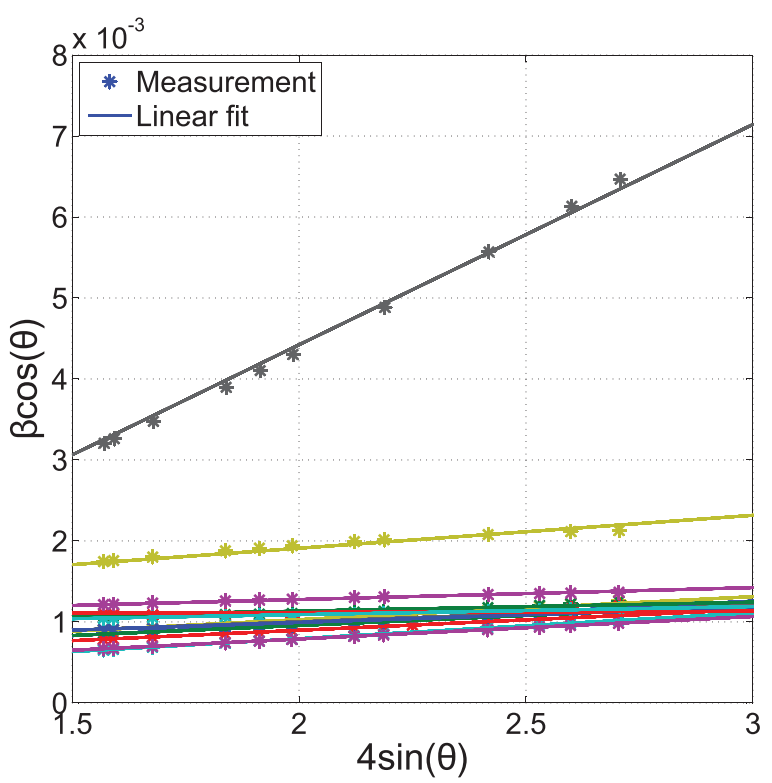

FIG. 3. Williamson-Hall plot of the ferromagnetic phase of $x=0.06$ for different temperatures. 


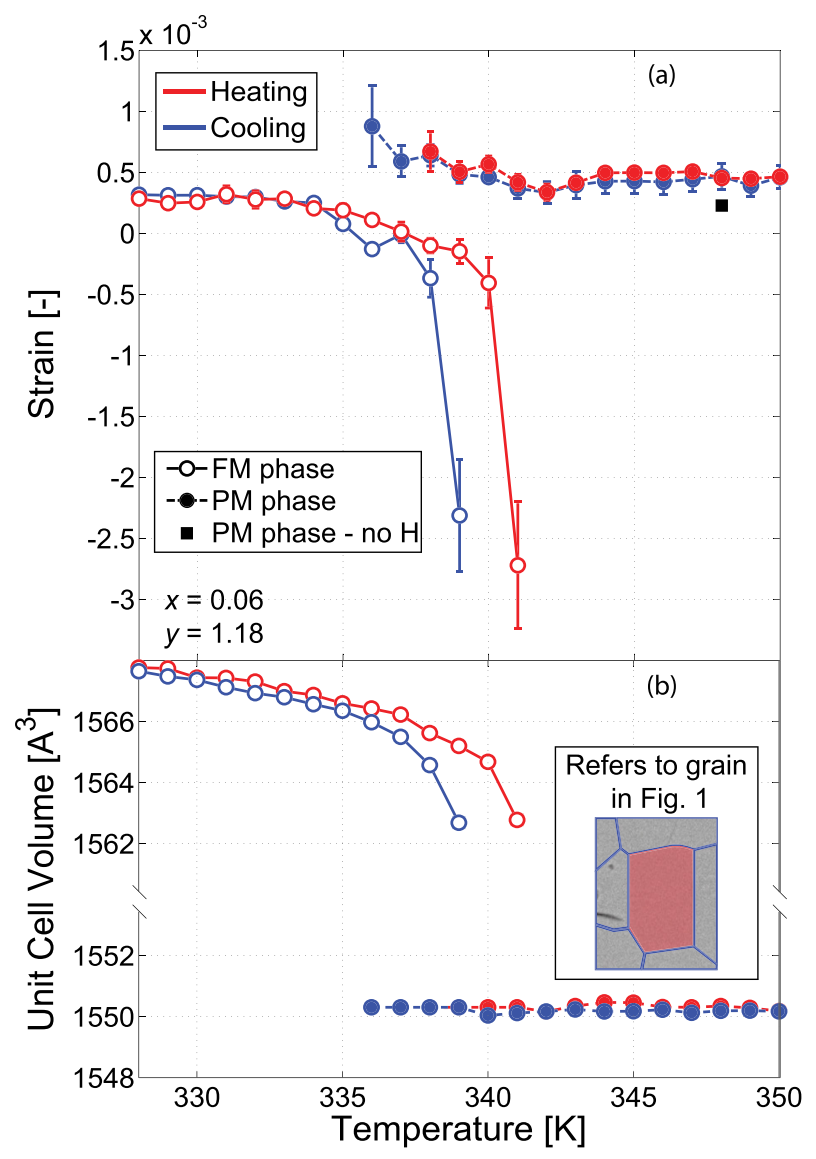

FIG. 4. (a) Strain and (b) volume as a function of the temperature. The inset shows a schematic micrograph of the material for explanation of the strain development during the transition.

to consider the different effects before, during, and after the transition in order to decide whether the strain is in fact positive or negative.

Figure 4(a) shows the strain and (b) the volume as a function of temperature for $x=0.06$. The error bars represent the error propagated from the different fits: Rietveld refinement fit of the $\mathrm{La}(\mathrm{Fe}, \mathrm{Mn}, \mathrm{Si}){ }_{13} \mathrm{H}_{z}$ material, the reference material $\mathrm{LaB}_{6}$ and the linear fit in Figure 3. The pure phases present some degree of non-uniform strain. This is expected as the material is imperfect - it contains some dislocation concentration and grain boundaries. Moreover, as shown previously, ${ }^{2}$ the material tends to exhibit chemical inhomogeneity that by itself could generate a non-uniform strain. In fact, the H-desorbed sample exhibits half of the strain of the hydrogenated sample, as shown in Figure 4. A possible annealing due to the hydrogenation process is ruled out, as the hydrogenation temperature is well below the polymorphic transformation, ${ }^{12}$ which in return leads to limited diffusion. Since hydrogen is inserted interstitially, which results in an increase of the unit cell, it is expected to generate positive strain on the pure phases.

The strain in the FM phase decreases to zero just below $T_{\mathrm{C}}$. This can be caused by the softening of the lattice induced by the magnetic interactions close to the transition. ${ }^{13}$ As can be seen in Figure 4(b), the FM volume decreases continuously with temperature. Therefore, it is most likely that compressive strain develops during the transition. Accordingly in Figures 4 and 5 , the strain during the transition is shown
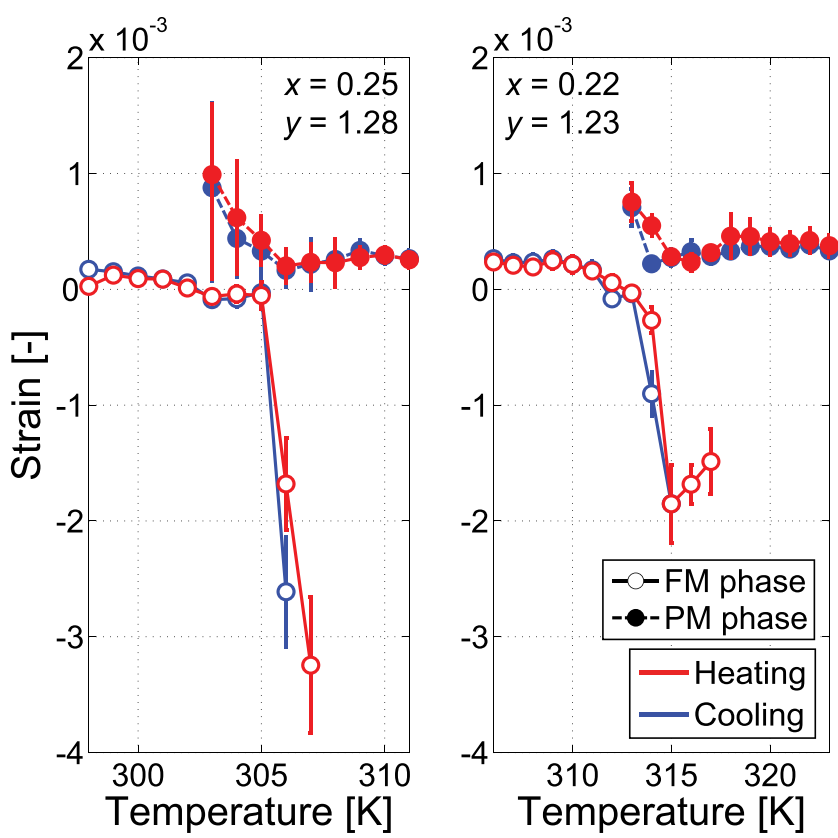

FIG. 5. Strain as a function of temperature for $\mathrm{x}=0.25$ and 0.22 , during heating and cooling.

with negative values. Figure 5 shows the strain development of two other FOPT $\mathrm{La}(\mathrm{Fe}, \mathrm{Mn}, \mathrm{Si}){ }_{13} \mathrm{H}_{z}$ compositions, showing a similar behavior, agreeing with what is observed for $x=0.06$.

The presence of chemical inhomogeneity (compositional variations) may be the reason behind the fact that the discontinuous FOPT is spread out across a range of temperatures and that strain develops as the phase transition proceeds. The inset in Figure 4(b) shows a grain of the main phase (highlighted in Figure 1). As the temperature increases, the grains in the FM phase become isolated and completely surrounded by PM phase grains. The PM phase, which is smaller, will then compress the FM phase. In addition to this reason for the occurrence of strain, the material is polycrystalline and even if there were no chemical inhomogeneity, strain development would be expected as the different crystallites have different orientation and irregular shapes. ${ }^{14}$

The FM and PM phases have different strain behavior. This can be related to the differing behavior of the bulk modulus of each phase, as observed in other magnetocaloric materials with a volume change at $T_{\mathrm{C}}$, e.g., $\mathrm{La}_{0.67} \mathrm{Ca}_{0.33} \mathrm{MnO}_{3}{ }^{15} \mathrm{~A}$ schematic drawing with an indication of the expected behavior of the bulk moduli is shown in Figure 6. The larger volume of the FM phase is attributed to the magneto-elastic coupling present in this material. As Gruner et al. ${ }^{13}$ have shown, close to the transition there is a softening of the lattice induced by the magnetic interactions. This will lead to a decrease of the bulk modulus of the FM phase, making this phase more susceptible to stresses through deformation. The same softening is not expected for the PM phase, where the magnetic moments are uncoupled; thus, the bulk modulus of this phase will be more or less constant close to the transition, as schematically shown in Figure 6.

The fracture mechanics of a multi-phase material such as $\left(\mathrm{La}(\mathrm{Fe}, \mathrm{Mn}, \mathrm{Si})_{13} \mathrm{H}_{z}, \alpha-\mathrm{Fe}\right.$ and La-rich phase) depends on 


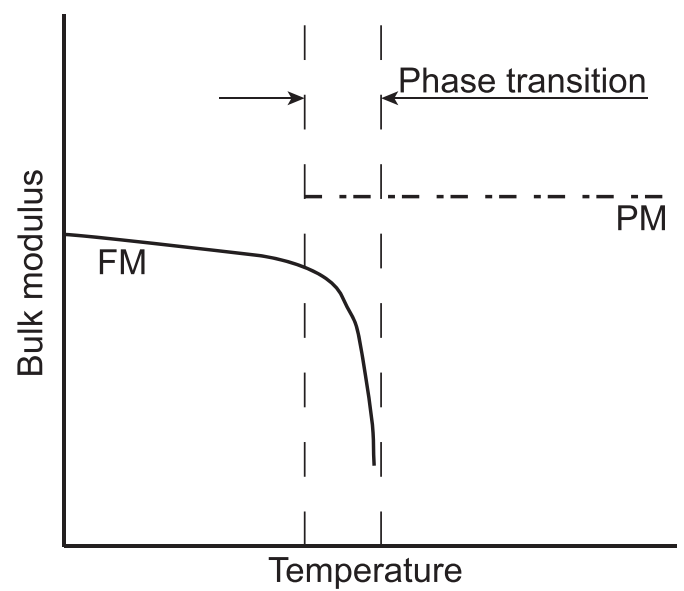

FIG. 6. Schematic drawing of the bulk moduli behavior of the ferromagnetic and paramagnetic phase.

the microstructure. It has been reported ${ }^{16}$ and also observed in the present investigation that $\alpha$-Fe crystallites are embedded within the crystallites of the main phase, while La-rich phases are located at the grain boundaries of the main phase. The latter, even if only present in such small amounts as to be undetectable by XRD, may play an important role in the cracking mechanism.

The cracking mechanism here proposed is analogous to the hydrogen-decrepitation observed in several rare-earth alloys. ${ }^{17-19}$ During the decrepitation process, hydrogen diffuses first in the rare-earth rich phase, expanding it and generating cracks. Upon further hydrogenation, the hydrogen also diffuses through the main phase (e.g., for Nd-magnets, $\mathrm{Nd}_{2} \mathrm{Fe}_{14} \mathrm{~B}$ ), which also breaks apart. The whole mechanism of hydrogen-decrepitation, i.e., powderization of a bulk material, is driven by the volume expansion of the different phases. In the case investigated here, an analogous process occurs. However, rather than being chemically driven, it is thermally/magnetically driven. Still, in this case we observe that the FM phase is significantly more susceptible to strain than the PM phase due to the softening of the magnetic interactions. Therefore, it seems that the cracking must start in the rare-earth-rich phase, which is also the only brittle phase without volume change, and then propagate throughout the sample; one can observe this behavior in Figure 1. In order to improve the mechanical stability of the material, this hypothesis should be further investigated. Additionally, we also believe that La-rich phase crystallites could work as nucleation sites for the transition, although further studies, e.g., with Hall-probe imaging, ${ }^{6}$ should be carried in order to confirm such an effect.

In conclusion, we have investigated the phase transition of $\mathrm{La}(\mathrm{Fe}, \mathrm{Mn}, \mathrm{Si})_{13} \mathrm{H}_{z}$ by careful XRD measurements and refinement. We observed an asymmetric development of strain, which differs from the FM to the PM phase. The strain development is related to three main features: (i) chemical inhomogeneity, (ii) different changes in the bulk moduli of the different phases, and (iii) the polycrystallinity of the samples. The interplay between these features leads to the strain development observed, which is intrinsically related to the occurrence of cracking during the phase transition. We note that a better understanding of the mechanical properties of magnetocaloric materials with a first order phase transition is crucial for designing materials for magnetic cooling applications. In this context, the role of the La-rich phase in crack formation should be further investigated in order to improve the mechanical stability of the material.

This work was financed by the ENOVHEAT project which is funded by Innovation Fund Denmark (Contract No 12-132673). The authors are grateful to Vacuumschmelze $\mathrm{GmbH}$ for providing the samples.

\footnotetext{
${ }^{1}$ A. Smith, C. R. H. Bahl, R. Bjørk, K. Engelbrecht, K. K. Nielsen, and N. Pryds, Adv. Energy Mater. 2, 1288-1318 (2012).

${ }^{2}$ H. N. Bez, K. K. Nielsen, P. Norby, A. Smith, and C. R. H. Bahl, AIP Adv. 6, 056217 (2016).

${ }^{3}$ S. Fujieda, A. Fujita, K. Fukamichi, Y. Yamazaki, and Y. Iijima, Appl. Phys. Lett. 79, 653 (2001).

${ }^{4}$ C. P. Bean and D. S. Rodbell, Phys. Rev. 126, 104-115 (1962).

${ }^{5}$ A. Waske, L. Giebeler, B. Weise, A. Funk, M. Hinterstein, M. Herklotz, K. Skokov, S. Fähler, O. Gutfleisch, and J. Eckert, Phys. Status Solidi RRL 9, 136-140 (2015).

${ }^{6}$ E. Lovell, A. M. Pereira, A. D. Caplin, J. Lyubina, and L. F. Cohen, Adv. Energy Mater. 5, 1401639 (2015).

${ }^{7}$ G. K. Williamson and W. H. Hall, Acta Metall. 1, 22-31 (1953).

${ }^{8}$ P. Scardi, M. Leoni, and R. Delhez, J. Appl. Crystallogr. 37, 381-390 (2004).

${ }^{9}$ M. Katter, V. Zellmann, G. W. Reppel, and K. Uestuener, IEEE Trans. Magn. 44, 3044-3047 (2008).

${ }^{10} \mathrm{M}$. André Meyers and K. K. Chawla, Mechanical Behavior of Materials, 2nd ed. (Cambridge University Press, 2009), p. 882.

${ }^{11}$ G. Caglioti, A. Paoletti, and F. P. Ricci, Nucl. Instrum. Methods 3, 223 (1958).

${ }^{12}$ K. Niitsu and R. Kainuma, Intermetallics 20, 160-169 (2012).

${ }^{13}$ M. E. Gruner, W. Keune, B. Roldan Cuenya, C. Weis, J. Landers, S. I. Makarov, D. Klar, M. Y. Hu, E. E. Alp, J. Zhao, M. Krautz, O. Gutfleisch, and H. Wende, Phys. Rev. Lett. 114, 057202 (2015).

${ }^{14}$ R. E. A. McKnight, T. Moxon, A. Buckley, P. A. Taylor, T. W. Darling, and M. A. Carpenter, J. Phys.: Condens. Matter 20, 075229 (2008).

${ }^{15}$ C. Zhu, R. Zheng, J. Su, and J. He, Appl. Phys. Lett. 74, 3504-3506 (1999).

${ }^{16}$ J. Liu, M. Krautz, K. Skokov, T. G. Woodcock, and O. Gutfleisch, Acta Mater. 59, 3602-3611 (2011).

${ }^{17}$ I. R. Harris, C. Noble, and T. Bailey, J. Less-Common Met. 106, L1-L4 (1985).

${ }^{18}$ M. Ragg, G. Keegan, H. Nagel, and I. R. Harris, Int. J. Hydrogen Energy 22, 333-342 (1997).

${ }^{19}$ H. N. Bez, C. S. Teixeira, B. G. F. Eggert, J. A. Lozano, M. S. Capovilla, J. R. Barbosa, Jr., and P. A. P. Wendhausen, IEEE Trans. Magn. 49,
} 4626-4629 (2013). 